Baseline

\title{
A metabolomic investigation of the effects of metal pollution in oysters Crassostrea hongkongensis
}

\author{
Chenglong Ji $\mathrm{i}^{\mathrm{a}}$, Qing Wang ${ }^{\mathrm{a}}$, Huifeng $\mathrm{Wu}^{\mathrm{a}, *}$, Qiaoguo Tan ${ }^{\mathrm{b}}$, Wen-Xiong Wang ${ }^{\mathrm{c}}$

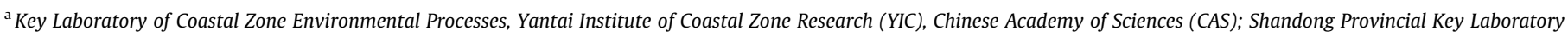 \\ of Coastal Zone Environmental Processes, YICCAS, Yantai 264003, PR China \\ ${ }^{\mathrm{b}}$ Key Laboratory of the Coastal and Wetland Ecosystems, Ministry of Education, College of Environment and Ecology, Xiamen University, Xiamen 361102, PR China \\ ${ }^{\mathrm{c}}$ Division of Life Science, The Hong Kong University of Science and Technology (HKUST), Clearwater Bay, Kowloon, Hong Kong
}

\section{A R T I C L E I N F O}

\section{Article history:}

Available online 12 December 2014

\section{Keywords:}

Metal pollution

Crassostrea hongkongensis

Gills

Metabolomics

${ }^{1} \mathrm{H}$ NMR

\begin{abstract}
A B S T R A C T
Metal pollution has been of great concern in the estuaries in Southern China. In this study, metabolic differences between oysters Crassostrea hongkongensis from clean and metal-polluted sites were characterized using NMR-based metabolomics. We collected oyster samples from one clean (Jiuzhen) and two metal polluted sites (Baijiao and Fugong). The metal concentrations in oyster gills indicated that both the Baijiao and Fugong sites were severely polluted by several metals, including $\mathrm{Cr}, \mathrm{Ni}, \mathrm{Cu}, \mathrm{Zn}, \mathrm{Ag}, \mathrm{Cd}$ and $\mathrm{Pb}$. In particular, $\mathrm{Cu}$ and $\mathrm{Zn}$ were the major contaminants from the Baijiao and Fugong sites. Compared with those oysters from the clean site (JZ), metal pollution in BJ and FG induced disturbances in osmotic regulation and energy metabolism via different metabolic pathways, as indicated by different metabolic biomarkers. This study demonstrates that NMR-based metabolomics is a useful tool for characterizing metabolic responses induced by metal pollution.
\end{abstract}

(c) 2014 Elsevier Ltd. All rights reserved.
Due to rapid industrialization and economic development, coastal metal pollution has become a threat to both coastal environments and organisms. Recent evidence has indicated that excessive contamination with various metals, such as $\mathrm{Cu}$, has caused oysters in the estuaries in Southern China to appear blue in color (Luo et al., 2014; Wang et al., 2014). This can be attributed to increasing anthropogenic activities in these rapidly developing areas. Therefore, a great deal of concern has been raised over the health risks to aquatic organisms and, subsequently, to humans from the consumption of seafood contaminated by high levels of metals (Wang et al., 2014). The excessive uptake of metals, including both non-essential (e.g., Cd) and essential (e.g., $\mathrm{Cu}$ ) metals, can induce serious toxic effects in aquatic organisms at multiple biological levels (e.g., biochemical, molecular, cellular, physiological) (Bertin and Averbeck, 2006; Cecconi et al., 2002).

Marine bivalves, including mussels, clams, cockles and oysters, are known to accumulate heavy metals in their tissues from metalpolluted environments (Goldberg et al., 1983) and are therefore used as good biomonitors and bioindicators for coastal metal pollution and ecotoxicology (Phillips and Segar, 1986). The oyster

\footnotetext{
* Corresponding author at: Key Laboratory of Coastal Zone Environmental Processes, Yantai Institute of Coastal Zone Research (YIC), Chinese Academy of Sciences (CAS), Yantai 264003, PR China. Tel.: +86 535 2109190; fax: +86 535 2109000.

E-mail address: hfwu@yic.ac.cn (H. Wu).
}

Crassostrea hongkongensis has shown its capability to accumulate various metals, such as $\mathrm{Cd}, \mathrm{Zn}$, and $\mathrm{Cu}$, to significant levels in its tissues (Liu and Wang, 2012). Similar to mussels and clams, oysters meet most of the requirements (wide geographical distribution, easy collection, high accumulation of metals and sessile lifestyle) for classification as sentinel organisms for metal monitoring (Luo et al., 2014; Liu and Wang, 2012). In addition, the oyster C. hongkongensis is an economically important species that is consumed in Southern China. Therefore, it is necessary to investigate the biological stress of metal pollution to this species.

Traditionally, to characterize biological stresses induced by metals, researchers may measure a few responses in selected biomarkers, such as lysosomal membrane stability, to test for the activation of catabolic processes, and anti-oxidative biochemical indices, to test for oxidative stress (Rank et al., 2007; de Almeida et al., 2004; Cappello et al., 2013; Regoli, 2000). One way to obtain a global view of the biological responses induced by metal pollution is to employ systems biology approaches, which have greatly expanded the biomarker discovery approaches for the global analysis of molecules in a biological system. System biology contains several -omic techniques including genomics, transcriptomics, proteomics and metabolomics (Ji et al., 2013; Santos et al., 2010; Williams et al., 2009). These -omic approaches have demonstrated their capabilities to discover broader ranges of molecular biomarkers (Katsiadaki et al., 2010; Williams et al., 2011; Liu et al., 2011; Zhang et al., 2011). Among these -omics techniques, 
metabolomics focuses on the small molecular products of metabolism (<1000 Da) (Jones et al., 2008; Viant et al., 2003). Therefore, metabolomics can detect differences in the metabolome profile in response to environmental stressors (Cappello et al., 2013; Fasulo et al., 2012; Kwon et al., 2012). It has also been widely applied in multiple areas such as food quality (Tarachiwin et al., 2008), ecotoxicology (Jones et al., 2008) and plant sciences (Kim et al., 2010). Cappello et al. (2013) successfully identified metabolic responses to environmental pollution, primarily from exposure to $\mathrm{Hg}$ and polyaromatic hydrocarbons (PAHs), in the mussel Mytilus galloprovincialis caged in anthropogenic-impacted and reference sites along the Augusta coastline (Sicily, Italy). The metabolic disturbances in the gills of caged mussels $M$. galloprovincialis in the contaminated field site suggested that these mussels had suffered from adverse environmental conditions. To characterize the biological effects of heavy metal pollution, Kwon et al. (2012) employed NMR-based metabolomics to compare the metabolic profiles in marine mussels (Mytilus edulis) that were sampled from a heavy metal-polluted area (Onsan Bay) and a clean area (Dokdo area). The results demonstrated the presence of significant metabolic responses induced by heavy metal contamination and the applicability of NMR-based metabolomics for characterizing heavy metal contamination-induced biological effects.

In this study, NMR-based metabolomics was used to investigate metabolic responses in the oyster (Crassostrea hongkongensis) to metal pollution. Hong Kong oysters $C$. hongkongensis were collected from three estuary sites (Baijiao, Fugong and Jiuzhen). Among these sampling sites, Baijiao and Fugong were contaminated by different concentrations of metals (e.g., zinc, copper, manganese lead), and Jiuzhen was a relatively clean site that was used as a reference. The gills of the oyster $C$. hongkongensis were examined for their metabolic profiles because gills of marine bivalves are the first organ to suffer from metal pollution due to their large surface constantly exposed to seawater (de Oliveira et al., 2008). The aims of this study were to use NMR-based metabolomics to determine the metabolic responses and hence biological effects in gills from oysters $C$. hongkongensis exposed to metal pollution.
During the low tides on December 18, 2012 (Fig. 1), contaminated oysters $C$. hongkongensis were collected from the Baijiao (BJ, 24 $28^{\prime} 2^{\prime \prime} \mathrm{N}, 117^{\circ} 56^{\prime} 19^{\prime \prime} \mathrm{E}$ ) and Fugong (FG, 24⒉ $22^{\prime} 58^{\prime \prime} \mathrm{N}$, $117^{\circ} 54^{\prime} 13^{\prime \prime}$ E) sites, along the contaminated Jiulongjiang estuary, and the control oysters were collected from the harbor of the Jiuzhen (JZ, 24 ${ }^{\circ} 2^{\prime} 38^{\prime \prime} \mathrm{N}, 117^{\circ} 42^{\prime} 26^{\prime \prime} \mathrm{E}$ ) site, from the nearby relatively clean Jiuzhen estuary, Fujian, China,. Evidence confirmed that the Jiulongjiang estuary was contaminated by metals (Liu and Wang, 2012; Zhang et al., 2007). This estuary is an important area for oyster culture, and the average surface salinity ranges from 14 to $26 \mathrm{psu}$ depending on the tidal action (Liu and Wang, 2012). Ten individual oysters of similar sizes were sampled from each site, and the gills were immediately dissected and flash frozen in liquid $\mathrm{N}_{2}$. After transport to our laboratory, these oyster samples were stored at $-80^{\circ} \mathrm{C}$ before metabolite extraction and metal analysis. All of the practical procedures for oyster sampling were strictly performed according to the guidelines suggested by Hines et al. (2007) and Vidal-Liñán and Bellas (2013).

Polar metabolites were extracted from the gill tissues of oysters by a modified extraction protocol using methanol/chloroform (Zhang et al., 2011). Briefly, the gill tissue (approx. $100 \mathrm{mg}$ ) was homogenized using a high-throughput homogenizer (Precellys 24, Bertin Technologies, France) and extracted in $4 \mathrm{~mL} / \mathrm{g}$ of methanol, $0.85 \mathrm{~mL} / \mathrm{g}$ of water and $2 \mathrm{~mL} / \mathrm{g}$ of chloroform. The mixture was shaken and centrifuged $\left(5 \mathrm{~min}, 3000 \mathrm{~g}\right.$, at $4{ }^{\circ} \mathrm{C}$ ), and the supernatant substance was removed. A total of $2 \mathrm{~mL} / \mathrm{g}$ of chloroform and $2 \mathrm{~mL} / \mathrm{g}$ of water were added to the supernatant, and the mixture was vortexed and then centrifuged again $\left(10 \mathrm{~min}, 3000 \mathrm{~g}, 4^{\circ} \mathrm{C}\right.$ ). The methanol/water layer, which contained polar metabolites, was transferred to a glass vial. The sample was dried in a centrifugal concentrator and stored at $-80^{\circ} \mathrm{C}$. It was subsequently re-suspended in $600 \mu \mathrm{L}$ of $100 \mathrm{mM}$ phosphate buffer $\left(\mathrm{Na}_{2} \mathrm{HPO}_{4}\right.$ and $\mathrm{NaH}_{2} \mathrm{PO}_{4}$ with $0.5 \mathrm{mM}$ TSP, $\mathrm{pH} 7.0$ ) in $\mathrm{D}_{2} \mathrm{O}$. The mixture was vortexed and then centrifuged at $3000 \mathrm{~g}$ for $5 \mathrm{~min}$ at $4{ }^{\circ} \mathrm{C}$. The supernatant substance $(550 \mu \mathrm{L})$ was then pipetted into a $5 \mathrm{~mm}$ NMR tube for NMR analysis.

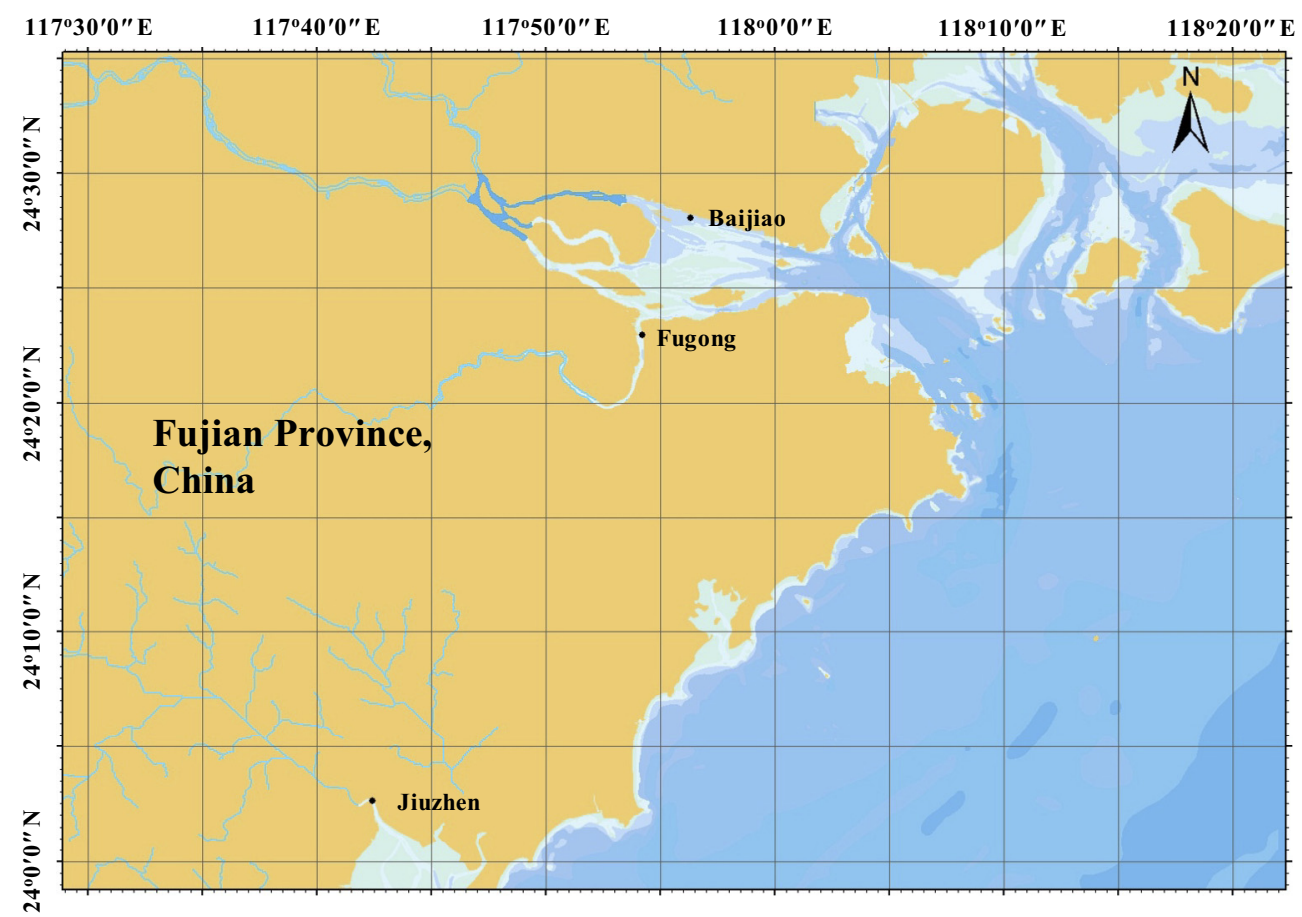

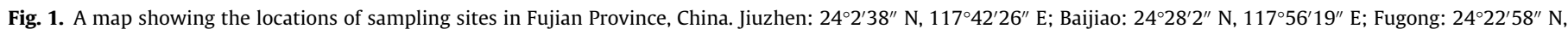

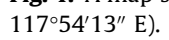


Extracts of gill tissue from oysters were analyzed on a Bruker AV 500 NMR spectrometer performed at $500.18 \mathrm{MHz}$ (at $298 \mathrm{~K}$ ) (Zhang et al., 2011). One-dimensional (1-D) ${ }^{1} \mathrm{H}$ NMR spectra were obtained using a $11.9 \mu$ s pulse, $6009.6 \mathrm{~Hz}$ spectral width, mixing time $0.1 \mathrm{~s}$, and 3.0 s relaxation delay with standard 1D NOESY pulse sequence, with 128 transients collected into 16,384 data points. Datasets were then zero-filled to 32,768 points, and exponential line-broadenings of $0.3 \mathrm{~Hz}$ were applied before Fourier transformation. All ${ }^{1} \mathrm{H}$ NMR spectra were phased, baseline-corrected, and calibrated (TSP at $0.0 \mathrm{ppm}$ ) manually using TopSpin (version 2.1, Bruker).

All one-dimensional ${ }^{1} \mathrm{H}$ NMR spectra were converted to a data matrix using the custom-written ProMetab software in MATLAB version 7.0 (MathWorks, Natick, MA, USA) (Viant et al., 2003). Each spectrum was segmented into bins with a width of $0.005 \mathrm{ppm}$ between 0.2 and $10.0 \mathrm{ppm}$. The bins of the residual water peak between 4.70 and $5.20 \mathrm{ppm}$ were excluded from all of the NMR spectra. The total spectral area of the remaining bins was normalized to unity to facilitate comparison among the spectra. All of the NMR spectra were subjected to a generalized log transformation with a transformation parameter of $\lambda=2.0 \times 10^{-8}$ to stabilize variance across the spectral bins and to increase the weightings of the less-intense peaks (Zhang et al., 2011). Data were meancentered before multivariate data analysis.

An unsupervised pattern recognition method principal component analysis (PCA) was used to reduce the dimensionality of the data and separate the groups of oyster samples from JZ, BJ and FG. A one-way analysis of variance (ANOVA) was conducted on the PC scores from each group to test the statistical significance $(P<0.05)$ of separations. Furthermore, the supervised multivariate data analysis methods, partial least squares discriminant analysis (PLS-DA) and orthogonal projection to latent structure with discriminant analysis (O-PLS-DA), were sequentially carried out to uncover and extract the statistically significant metabolite variations related to metal pollution. The results were visualized in terms of score plots to show the classifications and corresponding loading plots to show the NMR spectral variables contributing to the classifications. The model coefficients were calculated from the coefficients, incorporating the weight of the variables to enhance the interpretability of the model. Then, metabolic differences responsible for the classifications between control (JZ) and metal pollution-exposed (BJ or FG) groups could be detected in the coefficient-coded loading plots. The coefficient plots were generated using MATLAB with an inhouse developed program and were color-coded with the absolute values of coefficients (r). A hot color (i.e., red) corresponded to the metabolites with highly positive/negative significances in discriminating between groups, whereas a cool color (i.e., blue) corresponded to no significance. The correlation coefficient was determined based on the test for significance of the Pearson's product-moment correlation coefficient. Validation of the model was conducted using a 10 -fold cross validation, and the cross-validation parameter $Q^{2}$ was calculated. An additional validation method, the permutation test (permutation number $=200$ ), was conducted to evaluate the validity of the PLS-DA models. The $R^{2}$ value in the permutated plot described how well the data fit the derived model, whereas the $Q^{2}$ value described the predictive ability of the constructed model and provided a measure of the model's quality. If the maximum value of $Q^{2}$ from the permutation test was smaller than or equal to the $Q^{2}$ of the real model, the model was considered a predictable model. Similarly, the $R^{2}$ value and the difference between the $R^{2}$ and $Q^{2}$ values were used to evaluate the possibility of over-fitted models (Feng et al., 2013). Metabolites were assigned following the tabulated chemical shifts (Viant et al., 2003) and by the software Chenomx (Evaluation Version, Chenomx Inc., Edmonton, Alberta, Canada).

For metal analysis, each oyster tissue sample was dried to a constant weight and then digested in concentrated $\mathrm{HNO}_{3}$ at $80^{\circ} \mathrm{C}$ for $12 \mathrm{~h}$, after which a clear liquid was obtained. The metal concentration in the oyster samples was determined by inductively coupled plasma mass spectrometry (ICP-MS, Agilent 7700x). Appropriate internal standards ( $\mathrm{Sc}, \mathrm{Ge}, \mathrm{In}, \mathrm{Bi}$ ) were selected to correct for sensitivity drift and the matrix effect. A quality control sample was repeatedly measured after every 10 samples. The recovery of the analyzed metals from a standard reference material (SRM 1566b, oyster tissue) was within a $10 \%$ deviation from the certified values, except for $\mathrm{Cr}$, for which the certified concentration was not available. Metal concentrations were expressed as the mean \pm standard deviation (S.D.). All data concerning the metal concentrations were subjected to principal component analysis with autoscaling and Student's $t$-test. A $P$-value of less than 0.05 was considered significant. Statistical analysis was performed using Minitab software (Version 15, Minitab Inc. PA, USA).

The gill of bivalves is one of the major target organs for metal accumulation and detoxification (Liu and Wang, 2012; Wang et al., 2011). The concentrations ( $\mu \mathrm{g} / \mathrm{g}$ dry weight) of eight metals ( $\mathrm{Cr}, \mathrm{Ni}, \mathrm{Mn}, \mathrm{Cu}, \mathrm{Zn}, \mathrm{Ag}, \mathrm{Cd}$ and $\mathrm{Pb}$ ) and one metalloid (As) in gills from oysters $C$. hongkongensis collected from clean (JZ) and metal-contaminated (BJ and FG) sites are summarized in Table 1. Other metals/metalloids were present at similar concentrations in the gills (data not shown). To discern metal contamination situations, principal component analysis (PCA) was conducted to summarize the differences between different sampling sites, using the metal/metalloid concentrations as variables (Fig. 2). The biplot with samples (oyster samples from three sampling sites) and variables (eight metals and one metalloid) is shown in Fig. 2, with the first two principal components (PCs) containing $74.3 \%$ of the variance from the original nine dimensions. From a biplot with the samples and variables, each variable's contribution to the first two components for clustering the samples can be visualized directly. As shown in the biplot, these three groups of samples from JZ, BJ and FG were clearly separated along both the PC1 and PC2 axes, which demonstrated the significant differences in tissue metal concentrations between samples from these three sampling sites. Overall, along the PC1 axis, the oyster samples from both BJ and FG contained higher average levels of $\mathrm{Cu}, \mathrm{Zn}, \mathrm{Ag}, \mathrm{Ni}, \mathrm{As}, \mathrm{Cd}$, $\mathrm{Cr}$ and $\mathrm{Pb}$ than those from JZ. Specifically, $\mathrm{Cu}, \mathrm{Zn}, \mathrm{Ag}$ and As were distributed in the cluster of samples from BJ, which meant that the samples from BJ contained the highest average concentrations of these four metals/metalloid, with or without statistical significances (Table 1). In addition, the samples from FG contained the highest concentrations of $\mathrm{Cd}, \mathrm{Cr}$ and $\mathrm{Pb}$ because these three metals were located in the cluster of oyster samples from FG (Fig. 2). This

Table 1

Metal/metalloid concentrations in gill tissues from oysters Crassostrea hongkongensis sampled from three estuarine sites (JZ, BJ and FG).

\begin{tabular}{lccc}
\hline $\begin{array}{l}\text { Metal/metalloid } \\
\text { concentration } \\
(\mu \mathrm{g} / \mathrm{g} \mathrm{dw})^{\mathrm{a}}\end{array}$ & \multicolumn{3}{l}{ Sampling site } \\
\cline { 2 - 4 } & $\mathrm{JZ}$ & $\mathrm{BJ}$ \\
\hline $\mathrm{Cr}$ & $0.7 \pm 0.3$ & $8.4 \pm 2.5^{* *}$ & $14.0 \pm 3.1^{* *}$ \\
$\mathrm{Ni}$ & $10.2 \pm 3.1$ & $16.9 \pm 4.5$ & $17.1 \pm 5.6$ \\
$\mathrm{Mn}$ & $104.7 \pm 30.0$ & $70.7 \pm 24.6$ & $52.0 \pm 23.0$ \\
$\mathrm{Cu}$ & $205.3 \pm 118.9$ & $12923.5 \pm 7121.4^{* *}$ & $3792.0 \pm 1455.5^{* * *}$ \\
$\mathrm{Zn}$ & $3804.6 \pm 2403.8$ & $14912.6 \pm 6510.6^{*}$ & $9601.0 \pm 3664.7^{*}$ \\
$\mathrm{As}$ & $10.0 \pm 1.4$ & $12.4 \pm 3.1$ & $9.3 \pm 1.7$ \\
$\mathrm{Ag}$ & $1.4 \pm 0.7$ & $14.2 \pm 9.2^{*}$ & $3.4 \pm 1.7$ \\
$\mathrm{Cd}$ & $6.4 \pm 1.8$ & $10.1 \pm 3.1$ & $19.0 \pm 5.4^{* * *}$ \\
$\mathrm{~Pb}$ & $1.1 \pm 0.3$ & $1.8 \pm 0.5$ & $2.8 \pm 1.0^{*}$ \\
\hline
\end{tabular}

a Data are shown as the mean \pm standard deviation $(n=5)$. Values are presented as $\mu \mathrm{g} / \mathrm{g}$ dry weight.

$P<0.05$ indicate significant differences in metal concentrations between the clean (JZ) and metal-polluted sites (BJ or FG) (Student's $t$-test).

$P<0.01$ indicate significant differences in metal concentrations between the clean (JZ) and metal-polluted sites (BJ or FG) (Student's t-test). 


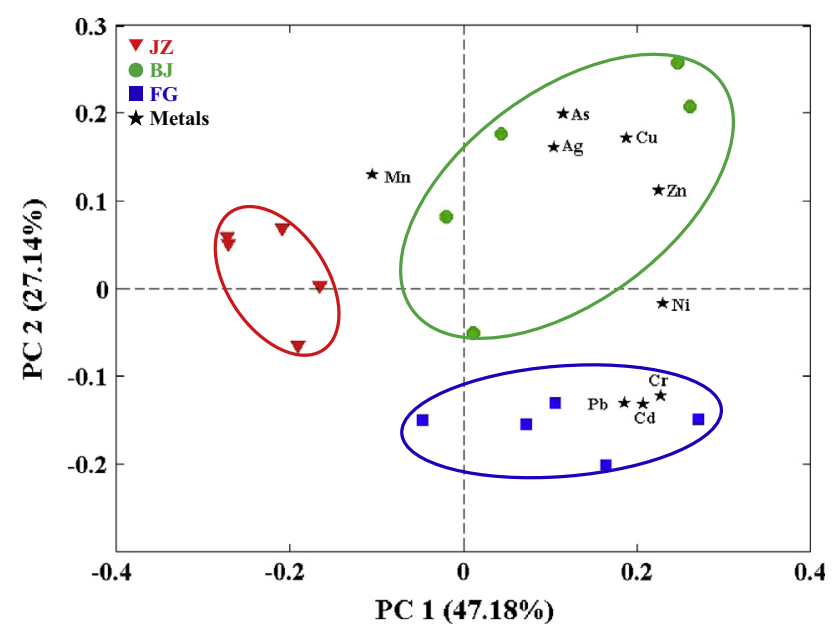

Fig. 2. The biplot containing the $P C$ scores for oyster samples from the three sampling sites, Jiuzhen (JZ, $\nabla$ ), Baijiao (BJ, O) and Fugong (FG, $\square$ ) and the variable (nine metals/metalloid) contributions for the clustering of oyster samples.

was confirmed by the tissue metal concentrations shown in Table 1. Obviously, both metal pollution-contaminated groups of samples had similar concentrations of $\mathrm{Ni}$ in the oyster gill tissues.

To compare the severity of metal pollution, an equation suggested by Liu and Wang (2012) was used to evaluate the integrated metal contamination:

Integrated metal contamination $=\sum_{i=0}^{m} C_{\text {contaminated }}^{i}-C_{\text {clean }}^{i}$

where $C_{\text {contaminated }}^{i}$ is the concentration of the $i$ th metal/metalloid in a contaminated oyster, and $C_{\text {clean }}^{i}$ is a reference value for the $i$ th metal/metalloid in an uncontaminated one. The reference values were determined from the clean JZ oysters. The variable $\mathrm{m}$ is the number of all metals/metalloids that an organism was simultaneously exposed to, i.e., $m=9$ in this work. Finally, we found that the oysters from the BJ site were the most severely contaminated ones, as indicated by the calculated integrated metal contamination in oyster gills. This is due to massive contributions to this term from the very high tissue concentrations of $\mathrm{Cu}$ and $\mathrm{Zn}$, which were approximately 60 and 4 times higher than those in the samples from the JZ site, respectively.

Representative ${ }^{1} \mathrm{H}$ NMR spectra of gill tissue extracts of oysters sampled from JZ, BJ and FG are shown in Fig. 3. Although several different classes of metabolites were identified, including amino acids (e.g., valine, leucine, isoleucine, alanine, arginine, glutamate, glutamine, aspartate, glycine), osmolytes (e.g., betaine, taurine, homarine, hypotaurine), energy storage compounds (e.g., glucose, glycogen) and Krebs cycle intermediates (succinate); all of the ${ }^{1} \mathrm{H}$ NMR spectra were dominated by organic osmolytes, particularly betaine and taurine, and the Krebs cycle intermediate, succinate. The identified metabolites are labeled in Fig. 3.

PCA was conducted on the ${ }^{1} \mathrm{H}$ NMR spectral datasets to summarize the similarities and differences between different sample groups. The PC scores plot presented significant $(P<0.01)$ separations between clean (JZ) and metal pollution-exposed groups (BJ and FG), along both the PC1 (65.23\% of variation) and PC2 (16.61\% of variation) axes (Fig. 4). Specifically, both metal pollution-exposed groups (BJ and $\mathrm{FG}$ ) were located along the negative part of the PC2 axis, which suggested that there were similar metabolic responses in these two groups to metal pollution. In addition, the oyster samples from the two metal pollution sites, BJ and FG, were located along the negative and positive PC1 axes, respectively (Fig. 4). This finding implied that there were also significantly different metabolic responses between the two metal pollution-exposed groups.

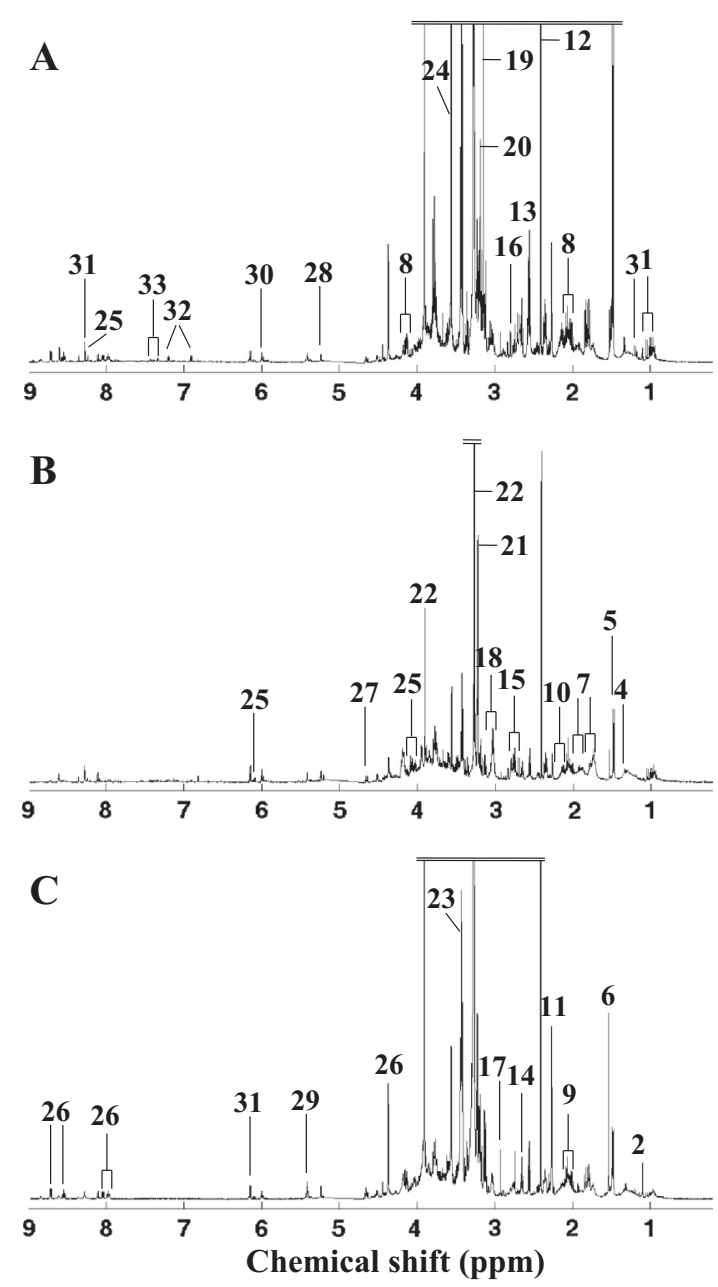

Fig. 3. Representative 1-dimensional $500 \mathrm{MHz}{ }^{1} \mathrm{H}$ NMR spectra of tissue extracts from gills of oysters $C$. hongkongensis from (A) Jiuzhen, (B) Baijiao and (C) Fugong sites. Keys: (1) branched chain amino acids: valine, leucine and isoleucine, (2) unknown 1 (1.10 ppm), (3) unknown 2 (1.16 ppm), (4) threonine, (5) alanine, (6) unknown 3 (1.50 ppm), (7) arginine, (8) proline, (9) glutamate, (10) glutamine, (11) acetoacetate, (12) succinate, (13) $\beta$-alanine, (14) hypotaurine, (15) aspartate, (16) dimethylamine, (17) dimethylglycine, (18) lysine, (19) malonate, (20) choline, (21) phosphocholine, (22) betaine, (23) taurine, (24) glycine, (25) AMP, (26) homarine, (27) $\beta$-glucose, (28) $\alpha$-glucose, (29) glycogen, (30) unknown 4 (5.98 ppm), (31) ATP, (32) tyrosine and (33) phenylalanine.

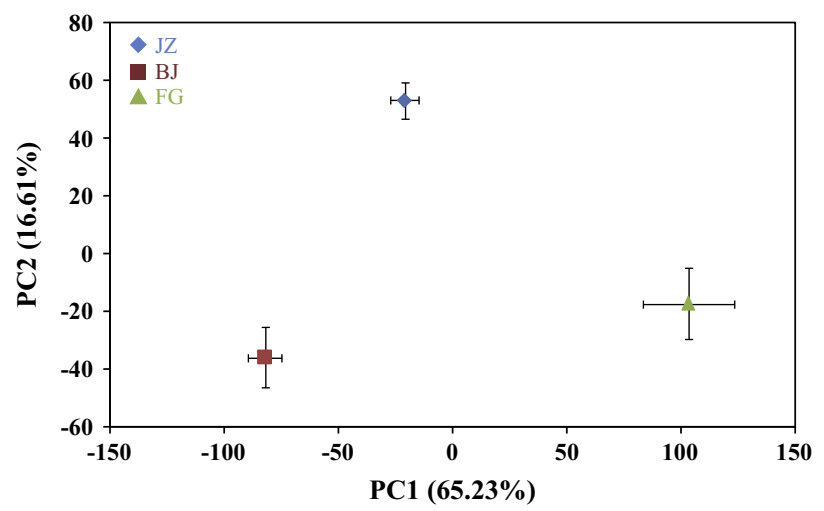

Fig. 4. Mean principal component analysis (PCA) scores plot of PC1 vs. PC2 for ${ }^{1} \mathrm{H}$ NMR spectra of tissue extracts of gills from oysters $C$. hongkongensis sampled from Jiuzhen $(\triangleleft)$ Baijiao $(\square)$ and Fugong $(\Delta)$ sites. The classes are presented as the mean \pm standard deviation (as associated standard error) of the PC scores for each group of oyster samples. 
Furthermore, O-PLS-DA was performed on the ${ }^{1} \mathrm{H}$ NMR spectral datasets from the control and metal pollution-exposed groups, respectively (Fig. 5). The score plots (Fig. 5A and C) derived from O-PLS-DA indicated clear classifications between control (JZ) and metal pollution-exposed groups, respectively, with reliable $Q^{2}$ values (>0.8). Based on the loading plot of O-PLS-DA (Fig. 5B), the oyster samples from BJ (compared with those from the clean site, JZ) were characterized by higher concentrations of branched chain amino acids (valine, leucine and isoleucine), arginine, aspartate, lysine, phosphocholine, proline, AMP and tyrosine and by lower concentrations of alanine, acetoacetate, $\beta$-alanine, betaine, malonate, taurine and homarine.

Compared with oyster samples from the control site (JZ), the metabolic profiles in the oyster samples from the FG site presented higher levels of arginine, glutamine, hypotaurine, aspartate, lysine, glucose, glycogen and AMP and lower levels of acetoacetate, $\beta$-alanine, betaine, malonate, taurine and homarine (Fig. 5D). Obviously, the common metabolic responses in both metal pollution-exposed groups included alterations in arginine, aspartate, lysine, AMP, acetoacetate, $\beta$-alanine, betaine, malonate, taurine and homarine. However, other metabolic responses such as BCAAs, phosphocholine, alanine, tyrosine, glutamine, hypotaurine, glucose and glycogen were uniquely altered in oyster samples from either BJ or FG.

Because the three investigated sampling sites (JZ, BJ and FG) were of similar water physico-chemical parameters (i.e., temperature, salinity, pH, dissolved oxygen) (Liu and Wang, 2012), the differential metabolic profiles should be related to the different metal pollutions that are present at the sampling sites. In the metal pollution-exposed oyster gills from both BJ and FG, three known osmolytes, i.e., betaine, taurine and homarine, were significantly lower than those in the oyster gills from the clean site (JZ), which suggested that metal pollution disturbed the osmotic regulation in oyster gills. Although the alteration patterns of amino acids in both metal pollution-exposed oyster gills were different, there were at least five amino acids with significantly higher levels in each metal pollution-exposed group than those in the uncontaminated oyster samples from JZ. Similar to betaine, taurine, homarine and hypotaurine, free amino acids are used in marine mollusks as osmolytes to balance their intracellular osmolarity with the environment (Viant et al., 2003; Kwon et al., 2012). Therefore, the markedly increased amino acids in metal pollution-exposed oyster gills were most likely used to compensate for the decreased osmolytes, betaine, taurine and homarine. Acetoacetate is a ketone body and can be synthesized from acetyl-coenzyme A (acetyl-CoA) as the end product of fatty acid metabolism. The low concentrations of acetoacetate implied that lipid metabolism is reduced in gills from metal pollution-exposed oysters. Malonate is a competitive inhibitor of the enzyme succinate dehydrogenase by binding to the active site of the enzyme without reacting. This finding suggested that metal pollution most likely affected the Krebs cycle related to energy metabolism in oysters.

For the oyster samples from the BJ site, the level of alanine was lower than that in the samples from the JZ site. In a previous study, Zhang et al. (2011) found that alanine was significantly decreased in Cu-exposed clams Ruditapes philippinarum and suggested that this decrease was a marker of disturbed osmotic regulation induced by $\mathrm{Cu}$. As a matter of fact, alanine is not only involved in anaerobic metabolism, but is another small organic osmolyte in marine bivalves (Preston, 1993). Interestingly, Cu was the most efficiently accumulated metal in oyster gills sampled from BJ. Therefore, the depleted alanine might be a unique metabolic biomarker of disturbed osmotic regulation induced by metal pollution, particularly by $\mathrm{Cu}$, in oyster gills from BJ. The increased AMP indicated enhanced the hydrolysis of ATP. Phosphocholine is made in a reaction that is catalyzed by choline kinase converting
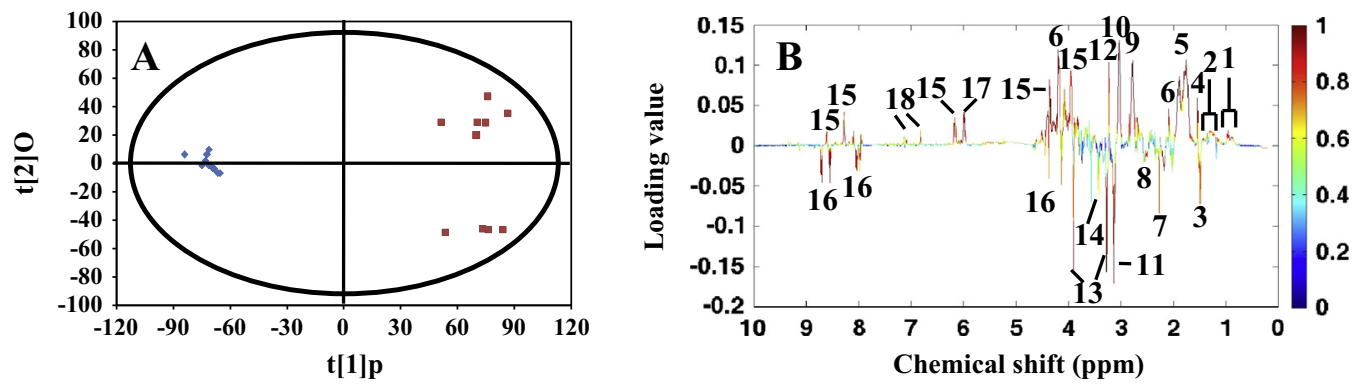

$R^{2} X=78.9 \%, Q^{2}=0.977$
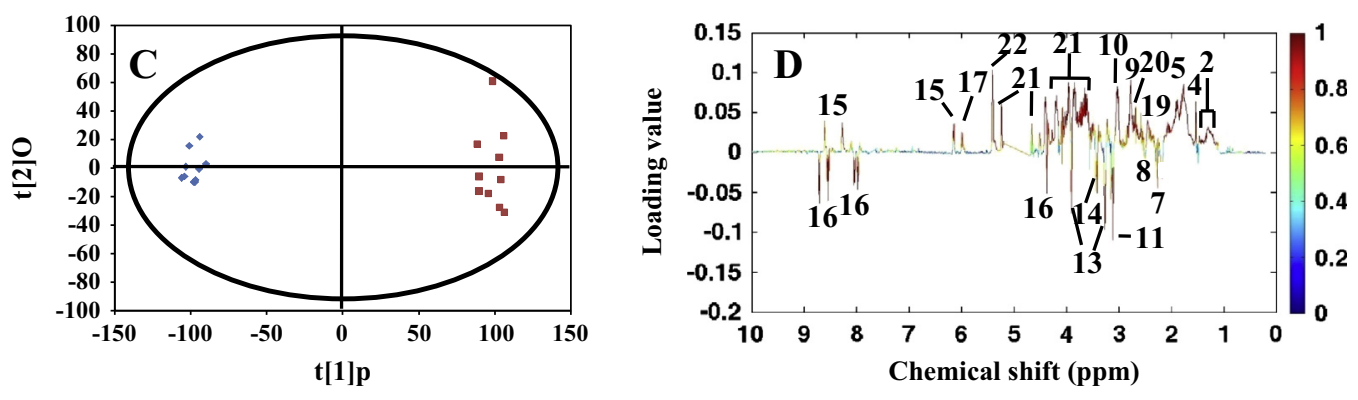

$R^{2} X=85.1 \%, Q^{2}=0.980$

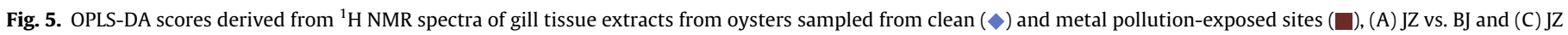

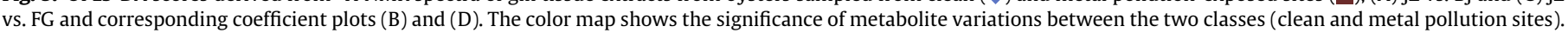

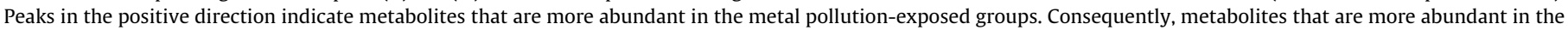

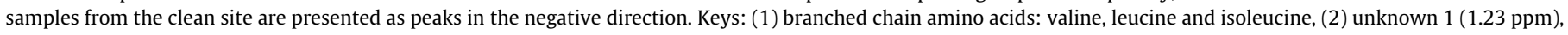

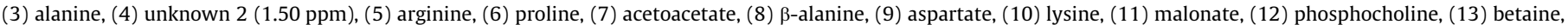

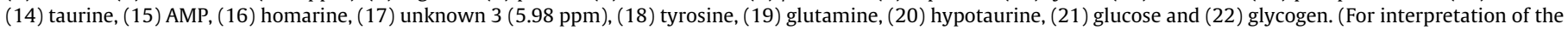
references to color in this figure legend, the reader is referred to the web version of this article.) 
ATP and choline into phosphocholine and ADP. Hence, the alterations in AMP and phosphocholine implied that there was a disturbance in energy metabolism induced by metal pollution in oyster samples from BJ.

Similar to the altered metabolic profiles in the oyster samples from BJ, their higher levels of amino acids (arginine, glutamine, $\beta$-alanine, aspartate, lysine) and hypotaurine and lower levels of betaine, taurine and homarine suggested that there may be disturbances in osmotic regulation induced by metal pollution in the oyster samples collected from FG. However, the higher level of AMP and lower levels of acetoacetate and malonate indicate a disturbance in energy metabolism, as noted above. The energy storage compounds, glucose and glycogen, were significantly higher in the samples from FG, which was different from those in BJ's samples. It might suggest that there is a reduced energy demand in oyster gills from BJ. Wu et al. (2013) observed an increased level of glycogen in the gill tissues from Cd-exposed clams Ruditapes philippinarum. In this work, interestingly, the oyster gills from FG accumulated more $\mathrm{Cd}(\sim 20$ times of that from JZ) than did those in oyster samples from BJ ( $\sim 12$ times that from JZ, Table 1$)$. Therefore, glycogen might be used as a biomarker for metal pollution, particularly for Cd, in FG.

As indicated by the concentrations of metals in oyster gills, both the Baijiao and Fugong sites were severely polluted by several metals, including $\mathrm{Cr}, \mathrm{Cu}, \mathrm{Zn}, \mathrm{Ag}, \mathrm{Cd}$ and $\mathrm{Pb}$. Among these metals, $\mathrm{Cu}$ and $\mathrm{Zn}$ were the major contaminants in both the Baijiao and Fugong sites. In this work, the metabolic differences between oysters Crassostrea hongkongensis from clean (Jiuzhen) and metal-polluted (Baijiao and Fugong) sites were characterized using NMR-based metabolomics. In summary, the metal pollution present at BJ and FG induced disturbances in osmotic regulation and energy metabolism via different metabolic pathways, as indicated by the different metabolic biomarkers found in oysters from these sites compared with those from the clean site, JZ. This study demonstrates that NMR-based metabolomics is a useful tool for characterizing the metabolic responses induced by metal pollution.

\section{Acknowledgments}

This work was supported by a Key Project from NSFC (21237004). We thank Prof. Mark Viant for the use of ProMetab software.

\section{References}

Bertin, G., Averbeck, D., 2006. Cadmium: cellular effects, modifications of biomolecules, modulation of DNA repair and genotoxic consequences (a review). Biochimie 88, 1549-1559.

Cappello, T., Maisano, M., D’Agata, A., Natalotto, A., Mauceri, A., Fasulo, S., 2013a. Effects of environmental pollution in caged mussels (Mytilus galloprovincialis). Mar. Environ. Res. 91, 52-60.

Cappello, T., Mauceri, A., Corsaro, C., Maisano, M., Parrino, V., Lo Paro, G., Messina, G., Fasulo, S., 2013b. Impact of environmental pollution on caged mussels Mytilus galloprovincialis using NMR-based metabolomics. Mar. Pollut. Bull. 77, 132-139.

Cecconi, I., Scaloni, A., Rastelli, G., Moroni, M., Vilardo, P.G., Costantino, L., Cappiello, M., Garland, D., Carper, D., Petrash, J.M., Del Corso, A., Mura, U., 2002. Oxidative modification of aldose reductase induced by copper ion. Definition of the metalprotein interaction mechanism. J. Biol. Chem. 277, 42017-42027.

de Almeida, E.A., Miyamoto, S., Bainy, A.C.D., de Medeiros, M.H.G., Mascio, P., 2004. Protective effect of phospholipid hydroperoxide glutathione peroxidase (PHGPX) against lipid peroxidation in mussels Perna perna exposed to different metals. Mar. Pollut. Bull. 49, 386-392.

de Oliveira, D.J.A., Salaroli, R.B., Fontanetti, C.S., 2008. Fine structure of Mytella falcata (Bivalvia) gill filaments. Micron 39, 329-336.

Fasulo, S., Iacono, F., Cappello, T., Corsaro, C., Maisano, M., D’Agata, A., Giannetto, A., de Domenico, E., Parrino, V., Lo Paro, G., Mauceri, A., 2012. Metabolomic investigation of Mytilus galloprovincialis (Lamarck 1819) caged in aquatic environments. Ecotoxicol. Environ. Saf. 84, 139-146.

Feng, J., Li, J., Wu, H., Chen, Z., 2013. Metabolic responses of HeLa cells to silica nanoparticles by NMR-based metabolomic analyses. Metabolomics 9, 874-886.
Goldberg, E.D., Koide, M., Hodge, V., Flegal, A.R., Martin, J., 1983. United States mussel watch - 1977-1978 results on trace metals and radionuclides. Est. Coast. Shelf Sci. 16, 69-93.

Hines, A., Oladiran, G.S., Bignell, J.P., Stentiford, G.D., Viant, M.R., 2007. Direct sampling of organisms from the field and knowledge of their phenotype: key recommendations for environmental metabolomics. Environ. Sci. Technol. 41, 3375-3381.

Ji, C., Wu, H., Wei, L., Zhao, J., Yu, J., 2013. Proteomic and metabolomic analysis reveal gender-specific responses of mussel Mytilus galloprovincialis to 2,2',4,4'tetrabromodiphenyl ether (BDE 47). Aquat. Toxicol. 140-141, 449-457.

Jones, O.A.H., Dondero, F., Viarengo, A., Griffin, J.L., 2008. Metabolic profiling of Mytilus galloprovincialis and its potential applications for pollution assessment. Mar. Ecol. Prog. Ser. 369, 169-179.

Katsiadaki, I., Williams, T.D., Ball, J.S., Bean, T.P., Sanders, M.B., Wu, H., Santos, E.M., Brown, M.M., Baker, P., Ortega, F., Falciani, F., Craft, J.A., Tyler, C.R., Viant, M.R., Chipman, J.K., 2010. Hepatic transcriptomic and metabolomic responses in the stickleback (Gasterosteus aculeatus) exposed to ethinyl-estradiol. Aquat. Toxicol. 97, 74-187.

Kim, H.K., Choi, Y.H., Verpoorte, R., 2010. NMR-based metabolomic analysis of plants. Nat. Proto. 5, 536-549.

Kwon, Y.K., Jung, Y.S., Park, J.C., Seo, J., Choi, M.S., Hwang, G.S., 2012. Characterizing the effect of heavy metal contamination on marine mussels using metabolomics. Mar. Pollut. Bull. 64, 1874-1879.

Liu, F., Wang, W.-X., 2012. Proteome pattern in oysters as a diagnostic tool for metal pollution. J. Hazard. Mat. 239-240, 241-248.

Liu, X., Zhang, L., You, L., Cong, M., Zhao, J., Wu, H., Li, C., Liu, D., Yu, J., 2011. Toxicological responses to acute mercury exposure for three species of Manila clam Ruditapes philippinarum by NMR-based metabolomics. Environ. Toxicol. Pharmacol. 31, 323-332.

Luo, L., Ke, C., Guo, X., Shi, B., Huang, M., 2014. Metal accumulation and differentially expressed proteins in gill of oyster (Crassostrea hongkongensis) exposed to longterm heavy metal-contaminated estuary. Fish Shellfish Immunol. 38, 318-329.

Phillips, D.J.H., Segar, D.A., 1986. Use of bioindicators in monitoring conservative contaminants: programme design imperatives. Mar. Pollut. Bull. 17, 10-17.

Preston, R.L., 1993. Transport of amino-acids by marine-invertebrates. J. Exp. Zool. 265, 410-421.

Rank, J., Lehtonen, K.K., Strand, J., Laursen, M., 2007. DNA damage, acetylcholinesterase activity and lysosomal stability in native and transplanted mussels (Mytilus edulis) in areas close to coastal chemical dumping sites in Denmark. Aquat. Toxicol. 84, 50-61.

Regoli, F., 2000. Total oxyradical scavenging capacity (TOSC) in polluted and translocated mussels: a predictive biomarker of oxidative stress. Aquat. Toxicol $50,351-361$.

Santos, E., Ball, J.S., Williams, T.D., Wu, H., Ortega, F., Van Aerle, R., Katsiadaki, I. Falciani, F., Viant, M.R., Chipman, J.K., Tyler, C.R., 2010. Identifying health impacts of exposure to copper using transcriptomics and metabolomics in a fish model. Environ. Sci. Technol. 44, 820-826.

Tarachiwin, L., Masako, O., Fukusaki, E., 2008. Quality evaluation and prediction of Citrullus lanatus by ${ }^{1} \mathrm{H}$ NMR-based metabolomics and multivariate analysis. J. Agric. Food Chem. 56, 5827-5835.

Viant, M.R., Rosenblum, E.S., Tjeerdema, R.S., 2003. NMR-based metabolomics: a powerful approach for characterizing the effects of environmental stressors on organism health. Environ. Sci. Technol. 37, 4982-4989.

Vidal-Liñán, L., Bellas, J., 2013. Practical procedures for selected biomarkers in mussels, Mytilus galloprovincialis Implications for marine pollution monitoring. Sci. Total Environ. 461-462, 56-64.

Wang, W.-X., Yang, Y., Guo, X., He, M., Guo, F., Ke, C., 2011. Copper and zinc contamination in oysters: subcellular distribution and detoxification. Environ. Toxicol. Chem. 30, 1767-1774.

Wang, W.-X., Pan, K., Tan, Q., Luo, L., Simpson, S.L., 2014. Estuarine pollution of metals in China: science and mitigation. Environ. Sci. Technol. 48, 9975-9976.

Williams, T.D., Turan, N., Diab, A.M., Wu, H., Mackenzie, C., Bartie, K.L., Hrydziuszko, O., Lyons, B.P., Stentiford, G.D., Herbert, J.M., Abraham, J.K., Katsiadaki, I., Leaver, M.J., Taggart, J.B., George, S.G., Viant, M.R., Chipman, K.J., Falciani, F., 2011. Towards a system level understanding of non-model organisms sampled from the environment: a network biology approach. PLoS Comput. Biol. 7, 1-20.

Williams, T.D., Wu, H., Santos, E., Ball, J., Katsiadaki, I., Brown, M.M., Baker, P. Ortega, F., Falciani, F., Craft, J.A., Tyler, C.R., Chipman, J.K., Viant, M.R., 2009. Hepatic transcriptomic and metabolomic responses in the stickleback (Gasterosteus aculeatus) exposed to environmentally relevant concentrations of dibenzanthracene. Environ. Sci. Technol. 43, 6341-6348.

Wu, H., Ji, C., Wang, Q., Liu, X., Zhao, J., Feng, J., 2013. Manila clam Venerupis philippinarum as a biomonitor to metal pollution. Chin. J. Oceanol. Limnol. 31, 65-74.

Zhang, L., Liu, X., You, L., Zhou, D., Wang, Q., Li, F., Cong, M., Li, L., Zhao, J., Liu, D., Yu, J., Wu, H., 2011a. Benzo(a)pyrene-induced metabolic responses in Manila clam Ruditapes philippinarum by proton nuclear magnetic resonance $\left({ }^{1} \mathrm{H}\right.$ NMR) based metabolomics. Environ. Toxicol. Pharmacol. 32, 218-225.

Zhang, L., Liu, X., You, L., Zhou, D., Wu, H., Li, L., Zhao, J., Feng, J., Yu, J., 2011b. Metabolic responses in gills of Manila clam Ruditapes philippinarum exposed to copper using NMR-based metabolomics. Mar. Environ. Res. 72, 33-39.

Zhang, L., Ye, X., Feng, H., Jing, Y., Ouyang, T., Yu, X., Liang, R., Gao, C., Chen, W., 2007. Heavy metal contamination in western Xiamen Bay sediments and its vicinity, China. Mar. Pollut. Bull. 54, 974-982. 